\title{
NPC-EXs Alleviate Endothelial Oxidative Stress and Dysfunction through the miR-210 Downstream Nox2 and VEGFR2 Pathways
}

\author{
Hua Liu, ${ }^{1}$ Jinju Wang, ${ }^{2}$ Yusen Chen, ${ }^{3}$ Yanfang Chen, ${ }^{2}$ Xiaotang Ma, ${ }^{3}$ Ji C. Bihl, ${ }^{2}$ and Yi Yang ${ }^{1}$ \\ ${ }^{1}$ College of Health Science, Wuhan Sports University, Wuhan 430079, China \\ ${ }^{2}$ Department of Pharmacology \& Toxicology, Boonshoft School of Medicine, Wright State University, Dayton, OH 45435, USA \\ ${ }^{3}$ Guangdong Key Laboratory of Age-Related Cardiac and Cerebral Diseases, Institute of Neurology, Affiliated Hospital of Guangdong \\ Medical University, Zhanjiang 524000, China
}

Correspondence should be addressed to Ji C. Bihl; ji.bihl@wright.edu and Yi Yang; yangyi999999@yahoo.com

Received 27 January 2017; Revised 12 April 2017; Accepted 20 April 2017; Published 28 May 2017

Academic Editor: Jaideep Banerjee

Copyright (C) 2017 Hua Liu et al. This is an open access article distributed under the Creative Commons Attribution License, which permits unrestricted use, distribution, and reproduction in any medium, provided the original work is properly cited.

\begin{abstract}
We have demonstrated that neural progenitor cells (NPCs) protect endothelial cells (ECs) from oxidative stress. Since exosomes (EXs) can convey the benefit of parent cells through their carried microRNAs (miRs) and miR-210 is ubiquitously expressed with versatile functions, we investigated the role of miR-210 in the effects of NPC-EXs on oxidative stress and dysfunction in ECs. NPCs were transfected with control and miR-210 scramble/inhibitor/mimic to generate NPC-EXs ${ }^{\text {con }}, \mathrm{NPC}^{-E X s^{\mathrm{sc}}}$, NPC$\mathrm{EXs}^{\text {anti-miR-210 }}$, and NPC-EXs ${ }^{\mathrm{miR}-210}$. The effects of various NPC-EXs on angiotensin II- (Ang II-) induced reactive oxygen species (ROS) overproduction, apoptosis, and dysfunction, as well as dysregulation of Nox2, ephrin A3, VEGF, and p-VEGFR2/ VEGFR2 in ECs were evaluated. Results showed (1) Ang II-induced ROS overproduction, increase in apoptosis, and decrease in tube formation ability, accompanied with Nox2 upregulation and reduction of p-VEGFR2/VEGFR2 in ECs. (2) Compared to NPC-EXs ${ }^{\text {con }}$ or NPC-EXs ${ }^{\text {sc }}$, NPC-EXs ${ }^{\text {anti-miR-210 }}$ were less whereas NPC-EXs ${ }^{\text {miR-210 }}$ were more effective on attenuating these detrimental effects induced by Ang II in ECs. (3) These effects of NPC-EXs ${ }^{\text {anti-miR-210 }}$ and NPC-EXs ${ }^{\text {miR-210 }}$ were associated with the changes of miR-210, ephrin A3, VEGF, and p-VEGFR2/VEGFR2 ratio in ECs. Altogether, the protective effects of NPC-EXs on Ang II-induced endothelial injury through miR-210 which controls Nox2/ROS and VEGF/VEGFR2 signals were studied.
\end{abstract}

\section{Introduction}

Oxidative stress is well-known to play a critical role in a diverse array of cardiovascular disorders including hypertension, diabetic vasculopathy, hypercholesterolemia, and atherosclerosis [1-3]. Indeed, overproduction of reactive oxygen species (ROS) has been shown to induce dysfunction, proinflammatory, and apoptotic death of endothelial cells (ECs) [4-6]. Therefore, an efficient antioxidant defense system to prevent endothelial dysfunction is critical in the protection of vasculature.

Recently, neural crest stem cells have been reported to promote the survival of neurons under normal and oxidative stress conditions in a superoxide dismutase 2 mutant neuron cell line [7]. As one type of stem cells in the brain, neural progenitor cells (NPCs) residing in the subventricular zone contact the blood vessels and directly juxtapose to ECs [8]. The two types of cells could interact with each other through direct physical contact or through paracrine mechanisms with potentially different biological effects. As showed in our previous study [9], NPCs can decrease hypoxia/reoxygenationinduced ROS overproduction on ECs. However, the exact mechanism of NPCs against oxidative stress remains unclear. Exosomes (EXs), small vesicles secreted by most cells, are emerging as mediators for cell-cell communications. They can transfer the carried cargoes such as microRNAs (miRs) and proteins to distant/nearby cells and thereby modulate the recipient cell function [10-12]. Stem cell-derived EXs have been shown to convey the benefits of their parent cells [13-17]. For example, endothelial progenitor cell-derived vesicles can protect ECs against hypoxia/reoxygenation injury [16]. Mesenchymal stem cell-derived EXs can promote functional recovery and neurovascular plasticity after stroke in rats through miR-133 [13] and enhance cell survival in kidney 
injury [17]. More recently, our group demonstrated that endothelial progenitor cell-derived vesicles from healthy controls have protective effects on the function of endothelial progenitor cells from diabetic patients through their carried miR-126 [14]. In order to explore the mechanism of NPCs protecting ECs from oxidative stress, we investigated whether EXs released from NPCs (NPC-EXs) can protect ECs from oxidative stress and dysfunction in an Ang II-induced injury model.

miR-210 is ubiquitously expressed in a wide range of cells, such as inducible pluripotent stem cells and bone marrow stem cells, and has versatile functions [18]. It is reported that miR-210 is a crucial element of ECs in response to hypoxia which considerably influences the endothelial angiogenic capability [19]. More studies have demonstrated that miR-210 not only influences cell survival by targeting apoptotic genes [20-22] but also displays antioxidant effect by reducing mitochondrial ROS production [23-25]. Recently, Wang et al. reported that EXs derived from inducible pluripotent stem cells can deliver miR-210 to the cardiomyocytes and protect the cardiomyocytes against $\mathrm{H}_{2} \mathrm{O}_{2}$-induced oxidative stress [15]. Nevertheless, there is no study investigating whether miR-210 is involved in the protective effects of NPC-EXs on ECs against oxidative stress.

In this study, we illustrated whether miR-210 participates in the protective effects of NPC-EXs on attenuating Ang IIinduced ROS overproduction and dysfunction in ECs.

\section{Materials and Methods}

2.1. Cell Culture of NPCs and ECs. Human NPCs were purchased from ATCC $^{\circledR}$ (ATCC-BYS012; Manassas, VA, USA) and cultured according to the manufacturer's protocol. Briefly, NPCs were cultured in complete growth medium which includes DMEM/F12 supplemented with the growth kit for NPC expansion (ATCC ACS-3003; Manassas, VA, USA). Medium was changed every other day. Human brain ECs were purchased from Cell Systems (Kirkland, WA, USA) and cultured with CSC complete medium (Cell Systems) containing $10 \%$ serum, $2 \%$ human recombinant growth factors, and $0.2 \%$ antibiotic solution under standard cell culture conditions $\left(5 \% \mathrm{CO} 2,37^{\circ} \mathrm{C}\right)$. Medium was changed twice a week.

2.2. Overexpression of $m i R-210$ in NPCs. NPCs were expanded and used for transfection to up- or downregulate of miR-210 [19]. Briefly, the NPCs were cultured to 60$70 \%$ confluence and transfected with miR-210 mimic, miR210 inhibitor, or the scramble control (SC) (40 nM, Exiqon, Woburn, MA) by using lipofectamine 2000 (Invitrogen, Carlsbad, CA) for $24 \mathrm{hrs}$ according to the manufacturer's instruction. NPCs transfected with miR-210 SC, inhibitor, or mimic were denoted as NPCs ${ }^{\mathrm{sc}}$, NPCs ${ }^{\text {anti-miR-210 }}$, or NPCs ${ }^{\text {miR-210 }}$, respectively. NPCs cultured in complete culture medium served as control $\left(\mathrm{NPCs}^{\mathrm{con}}\right)$. The three types of NPCs were used for producing corresponding EXs. All transfections were carried out in triplicate.

2.3. Preparation and Collection of EXs Released from NPCs. The protocol for collecting EXs from serum-free culture medium has been reported in our previous study [26].
Briefly, NPCs ${ }^{\text {con }}, \mathrm{NPCs}^{\mathrm{sc}}, \mathrm{NPC} s^{\text {anti-miR-210 }}$, or NPCs ${ }^{\mathrm{miR}-210}$ were cultured in serum-free culture medium to release EXs which were denoted as NPC-EXs ${ }^{\text {con }}$, NPC-EXs ${ }^{\mathrm{sc}}$, NPC$\mathrm{EXs}^{\text {anti-miR-210, }}$ and NPC-EXs ${ }^{\mathrm{miR}-210}$. After $24 \mathrm{hrs}$, the medium was collected and centrifuged at $300 \mathrm{~g}$ for $15 \mathrm{mins}$ to remove dead cells. The supernatants were centrifuged at $2000 \mathrm{~g}$ for $30 \mathrm{mins}$ to remove cell debris, followed by centrifugation at $20,000 \mathrm{~g}$ for $70 \mathrm{mins}$ and ultracentrifugation at $170,000 \mathrm{~g}$ for $90 \mathrm{mins}$ to pellet EXs. The pelleted NPC-EXs ${ }^{\text {con }}$, NPC-EXs ${ }^{\text {sc }}$, NPC-EXs ${ }^{\text {anti-miR-210 }}$, or NPC-EXs ${ }^{\text {miR-210 }}$ were resuspended with phosphate-buffered saline (PBS) and aliquoted for nanoparticle tracking analysis (NTA) and coculture experiments. PBS was filtered through $20 \mathrm{~nm}$ filter (Whatman, Pittsburgh, PA).

2.4. Nanoparticle Tracking Analysis of NPC-EXs. The NanoSight NS300 (Malvern Instruments, Malvern, UK) was used to detect EXs as we previously reported [26]. Briefly, $700 \mu \mathrm{l}$ diluted suspensions containing NPC-EXs were loaded into the sample chamber and the camera level was maintained at 10 for light scatter mode for sample analysis. Three videos of typically 30 -second duration were taken, with a frame rate of 30 frames per second. Data was analyzed by NTA 3.0 software (Malvern Instruments, Malvern, UK) on a frame-byframe basis. The experiment was repeated four times.

2.5. Labeling of NPC-EXs. NPC-EXs were labeled with a red fluorescence dye PKH26 (Sigma Aldrich, St. Louis, MO) as we previously reported [16]. In brief, NPC-EXs were incubated with $2 \mu \mathrm{M}$ PKH 26 dye in PBS for 5 mins at RT. An equal volume of FBS was added to stop staining. Then, NPC-EXs were pelleted by ultracentrifugation and resuspended with culture medium for coculture experiments.

2.6. Ang II Injury Model in ECs. ECs were seeded in 12-well plates $\left(5 \times 10^{4}\right.$ cells/well) during the logarithmic growth phase. When $70-80 \%$ confluent was reached, the cells were incubated with Ang II ( 0 or $10^{-6} \mathrm{M}$; Sigma-Aldrich, St. Louis, MO) for $24 \mathrm{hrs}$ [27]. After coincubation, the medium was replaced with fresh culture medium. ECs were then used for coculture experiments or collected for various analyses.

2.7. Coculture of NPC-EXs with ECs. To further elucidate the effects NPC-EXs on ECs, ECs were divided into five coculture groups: vehicle (coculture medium only), NPC-EXs ${ }^{\text {con }}$, NPC$\mathrm{EXs}^{\mathrm{sc}}, \mathrm{NPC}-\mathrm{EXs}^{\text {anti-miR-210}}$, or NPC-EXs ${ }^{\mathrm{miR}-210}$. Briefly, the NPC-EXs labeled with PKH 26 were resuspended with CSC medium and added to the culture medium of ECs subjected to Ang II injury. The concentration of NPC-EXs $(50 \mu \mathrm{g} / \mathrm{ml})$ was determined based on our previous study [16]. After $24 \mathrm{hr}$ coculture, the incorporation of NPC-EXs into ECs was observed by fluorescence microscopy (EVOS; Thermo Fisher Scientific). The level of cellular fluorescence intensity was analyzed by ImageJ (NIH) according to the instruction and a previous report [28]. The culture medium of ECs was collected for measuring the concentration of VEGF by ELISA. ECs were used for apoptosis, tube formation, and ROS production assays. The levels of miR-210, ephrin A3, Nox2, and p-VEGFR2/VEGFR2 in ECs were analyzed by quantitative RT-PCR and Western blot, respectively. ECs 
cultured under normal condition were used as controls. The experiment was repeated for four times.

2.8. Apoptosis Assay of ECs. After coculture, ECs were detached for apoptosis assay by using the FITC Annexin V apoptosis detection kit (BD Biosciences, CA) [9]. Briefly, cells were washed with PBS, resuspended with $100 \mu \mathrm{L} 1 \mathrm{x}$ annexin-binding buffer, incubated with $5 \mu \mathrm{L}$ FITCconjugated annexin $\mathrm{V}$ and $5 \mu \mathrm{L}$ propidium iodide (PI) for 15 mins in the dark, and then analyzed by flow cytometry. The apoptotic cells were defined as annexin V+/PI- cells. The percentage of apoptotic cells was calculated as the following: annexin V+/PI- cells/total cells $\times 100 \%$. The experiment was repeated four times.

2.9. Measurement of ROS. Intracellular ROS production was determined by dihydroethidium (DHE) (Sigma) staining. Briefly, cells were incubated with $2 \mu \mathrm{M}$ DHE solution at $37^{\circ} \mathrm{C}$ for $2 \mathrm{hrs}$. Cells were then washed twice with PBS, and images were taken under a fluorescence microscope (EVOS, Life Sciences). The level of cellular fluorescence intensity was analyzed by ImageJ (NIH, Bethesda, MD) according to a previous report [29]. Data are expressed as fold of fluorescence compared with the control. ECs cultured with CSC medium were used as control.

2.10. Quantitative RT-PCR Analysis. After transfection, cells were washed twice with PBS. The miRs from NPCs and their released EXs, as well as the ECs cocultured with various NPC-EXs were extracted using mirVana miRNA isolation kit (Qiagen, Hilden, Germany). For detecting miR-210 level, reverse transcription (RT) reactions were performed by using mirVana qRT-PCR miRNA detection kit and hsa-miR-210 qRT-PCR primer set from Ambion. The RT primer was 5'GTCGTATCCAGTGCAGGGTCCGAGGTATTCGCACTG GATACGACGACTGT-3'. The forward primer of miR-210 was 5'-CACGCAGTCGTA TCCAGTGCAGG- ${ }^{\prime}$. The reverse primer of miR-210 was $5^{\prime}$-CCAGTGCAGGGTCCG AGG TA- $3^{\prime}$. The expression of U6 was used as endogenous control for each sample. The forward primer of U6 was $5^{\prime}$-CT CGCTTCGGCAGCACA-3', and the reverse primer of U6 was $5^{\prime}$-AACGCTTCACGAATTTGCGT-3'. Relative expression level of each gene was normalized to U6 and calculated using the $2^{-\Delta \Delta C T}$ method. The experiment was repeated four times.

2.11. Enzyme-Linked Immunosorbent Assay. The level of VEGF in the culture medium of ECs cocultured with NPC$\mathrm{EXs}^{\mathrm{con}}, \mathrm{NPC}-\mathrm{EXs}^{\mathrm{sc}}, \mathrm{NPC}-\mathrm{EXs}^{\text {anti-miR-210 }}$, or NPC-EXs ${ }^{\mathrm{miR}-210}$ were measured by enzyme-linked immunosorbent assay (ELISA) according to the manufacturer's instructions (R\&D Systems, Minneapolis, MN, USA). The concentration of VEGF was calculated as $\mathrm{pg} / \mathrm{ml}$ of culture medium. Each group was triplicated. The experiment was repeated four times.

2.12. The Tube Formation Assay for ECs. The tube formation assay was conducted by using in vitro angiogenesis assay kit (Chemicon, Rosemont, IL). First, the ECMatrix solution was thawed and mixed with the ECMatrix diluent. Then, the ECMatrix mixture was placed in a 96-well tissue culture
TABLE 1: NTA analysis of the size and concentration of various NPC-EXs.

\begin{tabular}{|c|c|c|}
\hline EX type & Size range $(\mathrm{nm})$ & $\begin{array}{c}\text { Concentration } \\
\left(\times 10^{6} \text { particles } / \mathrm{ml}\right)\end{array}$ \\
\hline NPC-EXs ${ }^{\text {con }}$ & $102 \pm 30$ & $5.67 \pm 0.07$ \\
\hline NPC-EXs ${ }^{\mathrm{sc}}$ & $104 \pm 43$ & $5.58 \pm 0.11$ \\
\hline NPC-EXs ${ }^{\text {anti-miR-210 }}$ & $100 \pm 51$ & $5.69 \pm 0.18$ \\
\hline NPC-EXs ${ }^{\text {miR-210 }}$ & $105 \pm 62$ & $5.74 \pm 0.08$ \\
\hline
\end{tabular}

NPC-EXs ${ }^{\text {con }}, \quad$ NPC-EXs $s^{\text {sc }}, \quad$ NPC-EXs ${ }^{\text {anti-miR-210 }}$, and NPC-EXs ${ }^{\text {miR-210 }}$ represent EXs released from NPCs cultured in culture medium (control) and transfected with scramble control, miR-210 inhibitor, or miR-210 mimic. Data are expressed as mean \pm SEM. $N=4$ /group.

plate at $37^{\circ} \mathrm{C}$ for $1 \mathrm{hr}$ to allow the matrix solution to solidify. ECs $\left(1 \times 10^{4}\right.$ cells/well $)$ were seeded onto the solidified matrix and incubated at regular cell culture conditions $(5 \% \mathrm{CO} 2$, $37^{\circ} \mathrm{C}$ ). After $24 \mathrm{hr}$ post-seeding, $2 \mu \mathrm{g} / \mathrm{ml}$ calcein (Fisher Scientific, Hampton, $\mathrm{NH}$ ) was directly added to the culture well and incubated for 20 mins prior to imaging under an inverted fluorescence microscope. Tubes were defined as a tube structure exhibiting a length 4 times of its width [30]. The number of tubes per field was determined. Five random microscopic fields were assessed in each well. The average number from the five fields represents a group.

2.13. Western Blot Analysis. After $24 \mathrm{hr}$ treatments, proteins of ECs in different groups were extracted with cell lysis buffer (Thermo Fisher Scientific, Waltham, MA) supplemented with complete mini protease inhibitor tablet (Roche, Basel, Switzerland). Then, the protein lysates were electrophoresed through SDS-PAGE gel and transferred onto PVDF membranes. The membranes were blocked with $5 \%$ nonfat milk for $1 \mathrm{hr}$ at room temperature and incubated with primary antibody against ephrin A3 (1:500; Abcam, Cambridge, MA), Nox2 (1:1000; Abcam, Cambridge, MA), VEGFR2 (1:1000; Abcam, Cambridge, MA), p-VEGFR2 (p-Flk1; $1: 1000$; Abcam, Cambridge, MA), or $\beta$-actin $(1: 4000$; Sigma, St. Louis, $\mathrm{MO}$ ) at $4^{\circ} \mathrm{C}$ overnight. On the next day, membranes were washed and incubated with horseradishperoxidase-conjugated anti-rabbit or anti-mouse IgG (1:40,000; Jackson Immuno Research Lab, West Grove, PA) for $1 \mathrm{hr}$ at room temperature. Blots were developed with enhanced chemiluminescence developing solutions, and images were quantified under ImageJ software. The experiment was repeated four times.

2.14. Statistical Analysis. All experiments were repeated for four times. Data are expressed as mean \pm SEM. Multiple comparisons were analyzed by one- or two-way ANOVA followed by LSD post hoc test. SPSS 17.0 statistical software was used for analyzing the data. For all measurements, a $p<0.05$ was considered statistically significant.

\section{Results}

3.1. Transfection of miR-210 Mimic and Inhibitor Altered the Level of miR-210 in NPCs and NPC-EXs. Upon the results obtained from NTA analysis (Table 1), modulation 


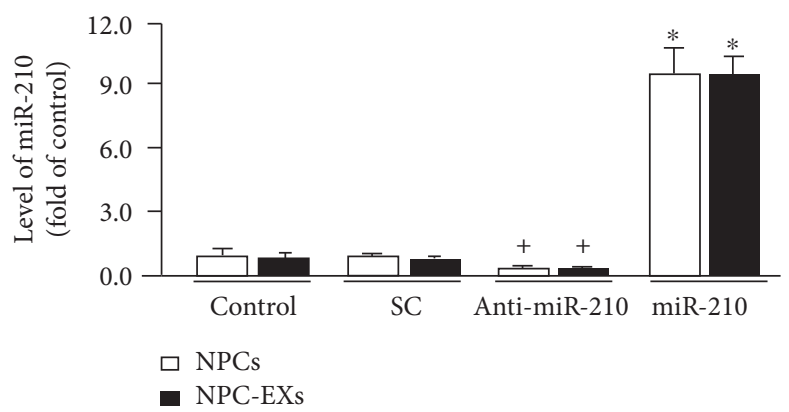

(a)
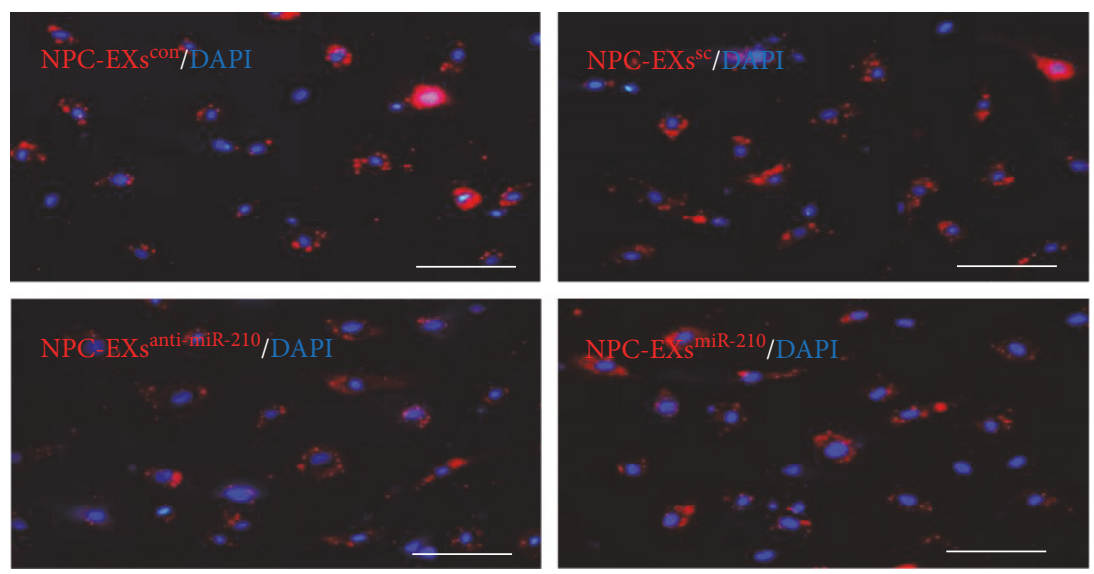

(b)

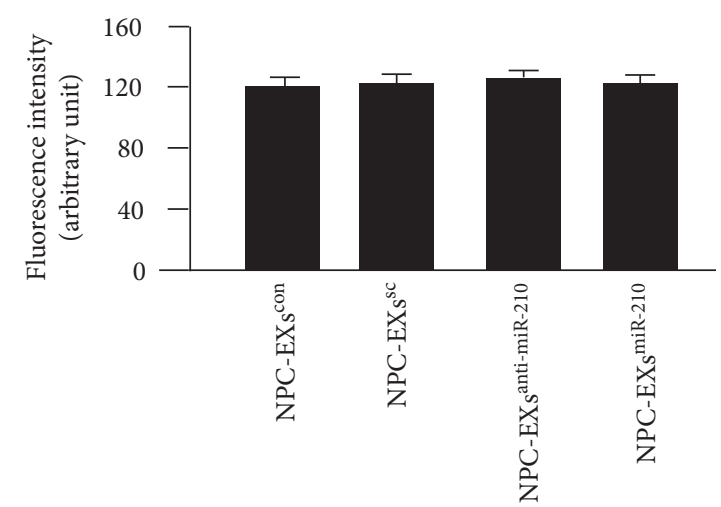

(c)

FIgURE 1: The levels of miR-210 in NPCs and their released NPC-EXs were changed by miR-210 mimic or inhibitor. (a) Summarized data showing the levels of miR-210 in NPCs and NPC-EXs. ${ }^{*} p<0.05$ versus control or SC or anti-miR-210; ${ }^{+} p<0.05$ versus control or SC. SC: scramble control of miR-210. (b) Representative images showing the incorporation of NPC-EXs into the cytoplasm of ECs. (c) Summarized data showing the fluorescence intensity in ECs. Data are expressed as mean \pm SEM. $N=4$ /group.

of miR-210 did not change the size and the concentration of EXs released from NPCs (versus NPC-EXs ${ }^{\text {con }}$ or NPC$\left.\mathrm{EXs}^{\mathrm{sc}}, p>0.05\right)$. As shown in Figure 1(a), transfection of the miR-210 mimic significantly increased miR-210 level in NPCs. As expected, miR-210 level was higher in NPC-EXs ${ }^{\text {miR-210 }}$ than that in NPC-EXs ${ }^{\text {con }}$ and NPC-EXs ${ }^{\text {sc }}$. On the contrary, miR-210 inhibitor significantly decreased the level of miR-210 in NPCs and their released EXs.

3.2. NPC-EXs ${ }^{\text {miR-210 }}$ Significantly Upregulated the Level of miR-210 and Downregulated Ephrin A3 Level in ECs. As shown in Figures 1(b) and 1(c), PKH26-labeled NPC-EXs were observed in the cytoplasm of ECs after $24 \mathrm{hr}$ of coculture, indicating EXs were uptaken by ECs. There was no significant difference of the fluorescence intensity among different groups.

In order to evaluate whether coculture with NPC-EXs can alter the level of miR-210 in ECs, we conducted quantitative RT-PCR to determine the level of miR-210 in ECs after different treatments. As shown in Figure 2(a), Ang II downregulated the level of miR-210 in ECs (versus control, $p<0.05)$. In the treatment groups, coculture with NPC-EXs ${ }^{\text {con }}$ or NPC-EXs ${ }^{\text {sc }}$ alone significantly upregulated miR-210 level in ECs (versus vehicle, $p<0.05)$. NPC-EXs ${ }^{\text {anti-miR-210 }}$ did not 


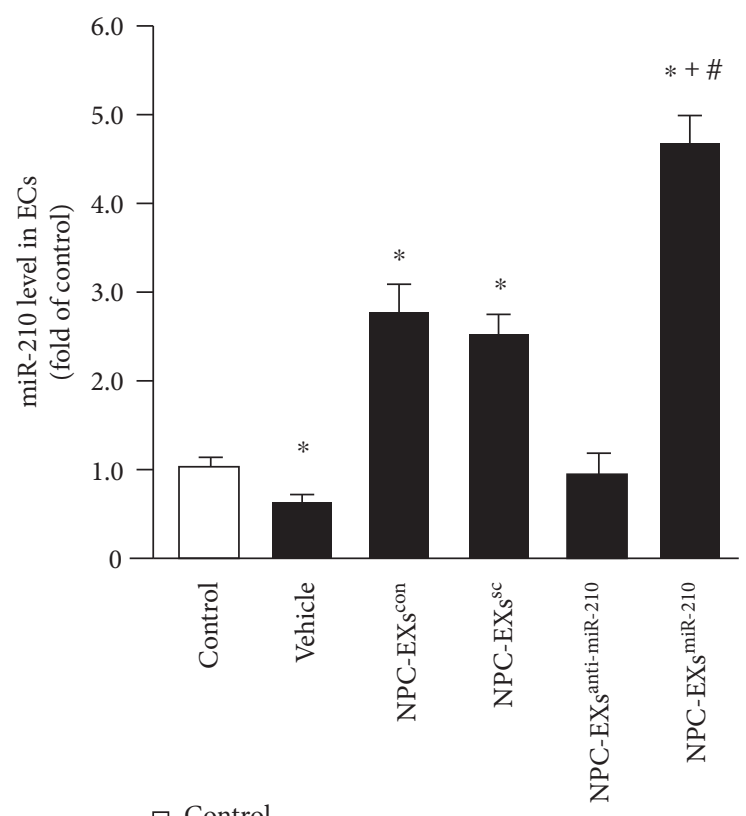

- Ang II

(a)

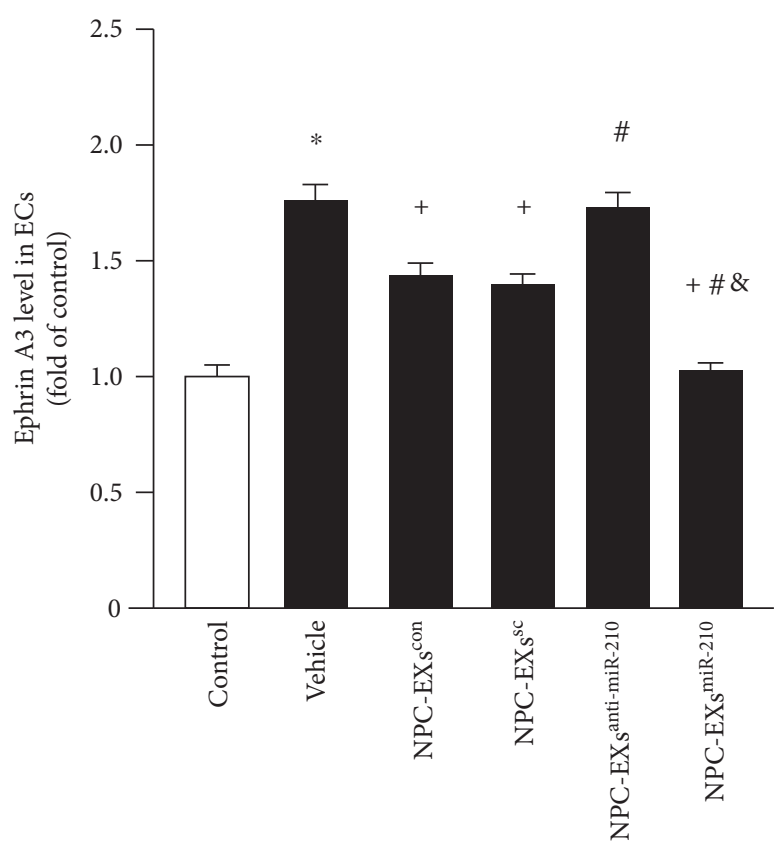

$\square$ Control

- Ang II

Ephrin A3
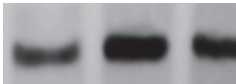

$\beta$-Actin

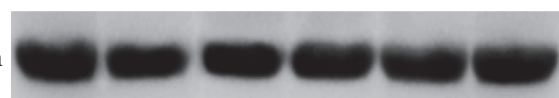

(b)

Figure 2: Up- or downregulation of miR-210 affected the effects of NPC-EXs on the levels of miR-210 and its target gene ephrin A3. (a) The level of miR-210 in ECs treated with various NPC-EXs. (b) Summarized data and representative bands showing the protein level of ephrin A3 in ECs treated with various NPC-EXs. ${ }^{*} p<0.05$ versus control; ${ }^{+} p<0.05$ versus vehicle; ${ }^{\#} p<0.05$ versus NPC-EXs ${ }^{\text {con }}$ or NPC-EXs $s^{\text {sc }} ;{ }^{\&} p<0.05$ versus NPC-EXs ${ }^{\text {anti-miR-210 }}$. EXs released from NPCs ${ }^{\text {con }}, \mathrm{NPCs}^{\text {sc }}, \mathrm{NPC}-\mathrm{EXs}^{\text {anti-miR-210 }}{ }^{\text {, or NPCs }}{ }^{\text {miR-210 }}$ were denoted as NPC-EXs ${ }^{\text {con }}, \mathrm{NPC}_{\mathrm{EXs}}{ }^{\mathrm{sc}}, \mathrm{NPC}-\mathrm{EXs}^{\text {anti-miR-210 }}{ }^{\text {, or NPC-EXs }}{ }^{\text {miR-210 }}$. Data are expressed as mean \pm SEM. $N=4 /$ group. 


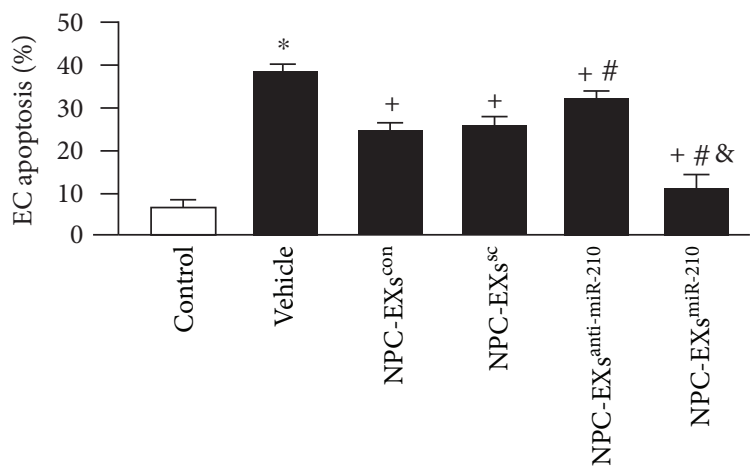

$\square$ Control

- Ang II

(a)
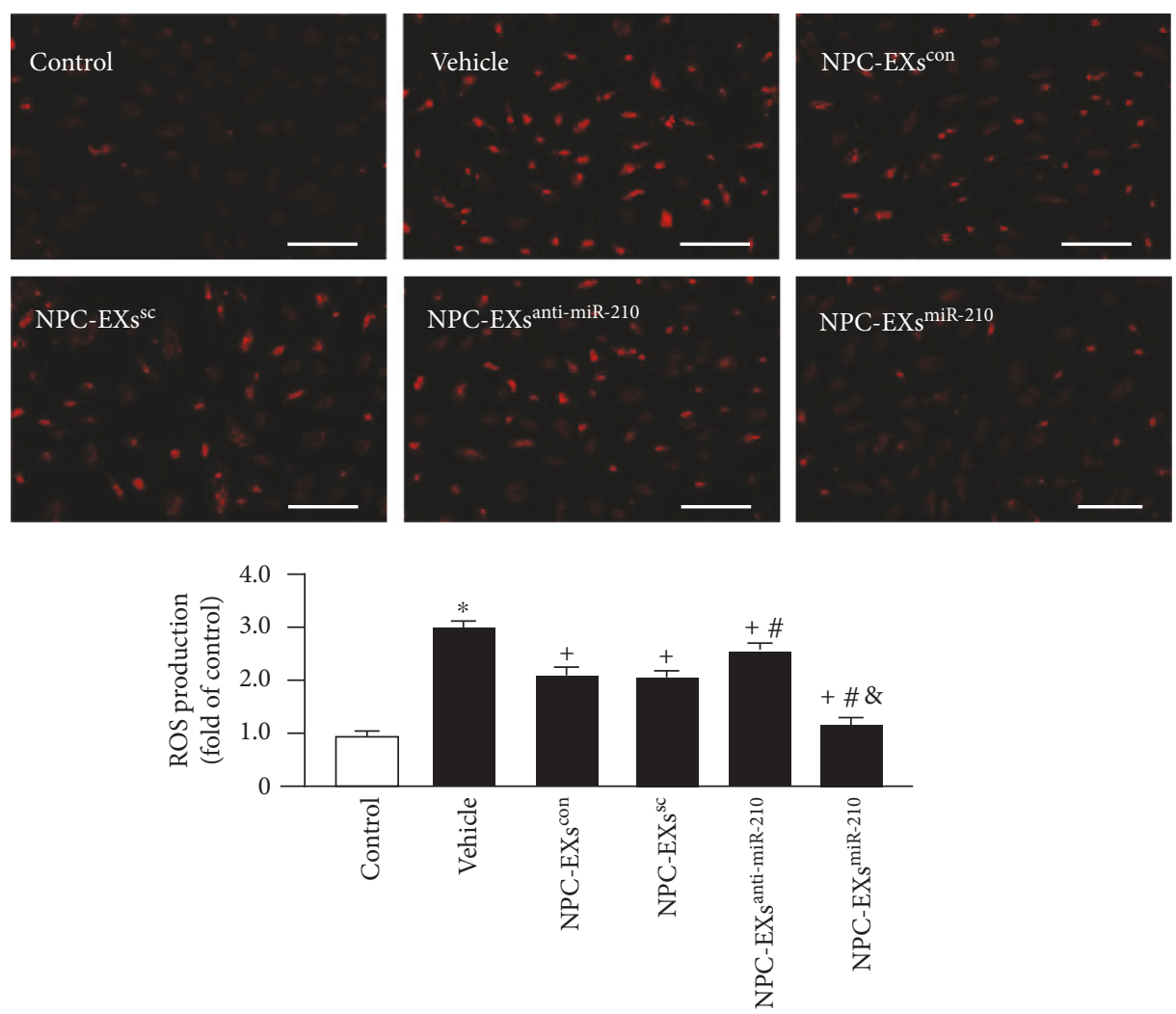

$\square$ Control

- Ang II

(b)

Figure 3: Up- or downregulation of miR-210 modulated the effects of NPC-EXs on reducing Ang II-induced apoptosis and ROS overproduction in ECs. (a) The apoptotic rate of ECs treated with different NPC-EXs. (b) Up: representative images showing DHE staining of ECs treated with different NPC-EXs; bar: $100 \mu \mathrm{m}$; bottom: summarized data; ${ }^{*} p<0.05$ versus con; ${ }^{+} p<0.05$ versus

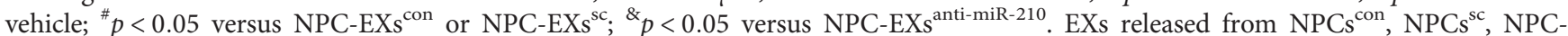

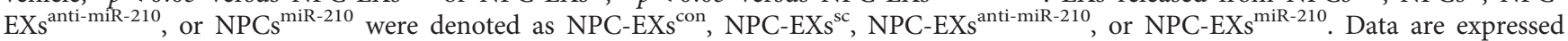
as mean \pm SEM. $N=4$ /group.

significantly alter the level of miR-210 in Ang II-injured ECs (versus vehicle, $p>0.05$ ), whereas, NPC-EXs ${ }^{\text {miR-210 }}$ remarkably raised the level of miR-210 in ECs (versus NPC-EXs ${ }^{\text {con }}$ or NPC-EXs ${ }^{\mathrm{sc}}$ or NPC-EXs $\left.{ }^{\text {anti-miR-210 }}, p<0.05\right)$. These data suggest that NPC-EXs can deliver miR-210 into ECs in vitro.
We also determined the protein level of ephrin A3, a target gene of miR-210, in ECs after coculture. Our data (Figure 2(b)) showed that the ephrin A3 level was upregulated in Ang II-injured ECs (versus control, $p<0.05$ ), which was downregulated by NPC-EXs ${ }^{\text {con }}$ or NPC-EXs ${ }^{\text {sc }}$ 


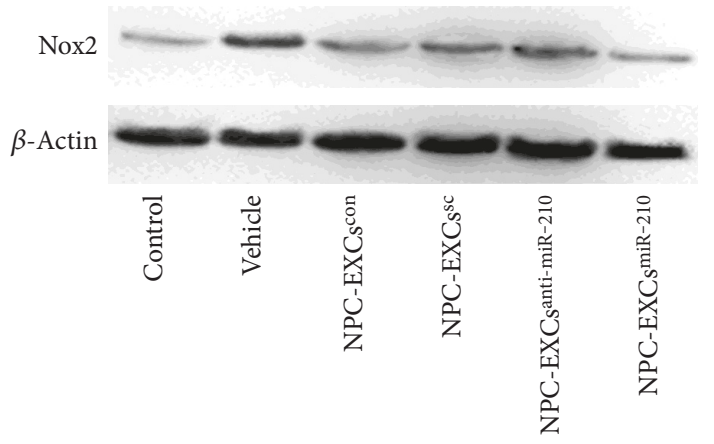

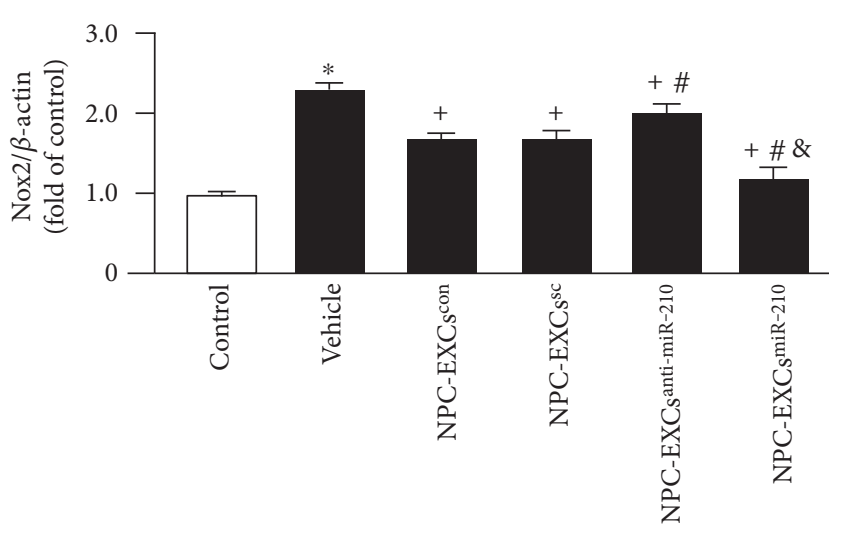

$\square$ Control

Ang II

Figure 4: Manipulation of miR-210 with inhibitor or mimic altered the effect of NPC-EXs on reducing Ang II-induced Nox2 expression. (a) Representative Western blot bands. (b) Summarized data showing the expression of Nox 2 in ECs treated with different NPC-EXs; ${ }^{*} p<0.05$ versus con; ${ }^{+} p<0.05$ versus vehicle; ${ }^{\#} p<0.05$ versus NPC-EXs ${ }^{\text {con }}$ or NPC-EXs ${ }^{\text {sc }} ;{ }^{\&} p<0.05$ versus NPC-EXs ${ }^{\text {anti-miR-210 }}$. EXs released from

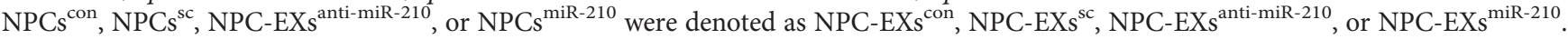
Data are expressed as mean \pm SEM. $N=4$ /group.

coculture (versus vehicle, $p<0.05$ ). NPC-EXs ${ }^{\text {anti-miR-210 }}$ did not significantly affect, while NPC-EXs ${ }^{\text {miR-210 }}$ upregulated the level of ephrin A3 in Ang II-injured ECs. These findings reflect that NPC-EXs and NPC-EXs ${ }^{\text {miR-210 }}$ could transfer miR-210 to ECs and modulate the expression of its target gene, ephrin A3, in ECs.

\subsection{NPC-EXs ${ }^{\text {anti-miR-210 }}$ Were Less, Whereas NPC-EXs ${ }^{\text {miR-210 }}$} Were More Effective on Reducing Ang II-Induced Apoptosis, ROS Overproduction, and Nox2 Upregulation on ECs. To verify the model of Ang II-induced EC injury, we conducted flow cytometry and DHE staining to evaluate the apoptotic rate and ROS production, respectively. Our results (Figure 3) showed that Ang II significantly increased the percentage of ECs in the early apoptosis phase and the level of ROS production (versus control, $p<0.05$ ).

To determine the effects of NPC-EXs on ECs against Ang II-induced oxidative stress injury, we cocultured Ang IIinjured ECs with various NPC-EXs. Our data showed that coculture with NPC-EXs ${ }^{\text {con }}$ or NPC-EXs ${ }^{\text {sc }}$ alone can reduce Ang II-induced apoptosis and ROS overproduction (versus vehicle, $p<0.05)$. NPC-EXs ${ }^{\text {anti-miR-210 }}$ can decrease these detrimental effects of Ang II, but was less than NPC-EXs ${ }^{\text {con }}$ and NPC-EXs ${ }^{\mathrm{sc}}$ did (versus NPC-EXs ${ }^{\text {con }}$ or NPC-EXs ${ }^{\mathrm{sc}}$, $p<0.05)$. NPC-EXs ${ }^{\mathrm{miR}-210}$ had the most effects on decreasing Ang II-induced apoptosis and ROS production of ECs among the four types of NPC-EXs.

In addition, we analyzed the expression of Nox 2 in ECs after cocultured with different NPC-EXs. Our Western blot results (Figure 4) showed that Ang II upregulated Nox2 expression in ECs (versus control, $p<0.05$ ). Coculture with NPC-EXs ${ }^{\text {con }}$ or NPC-EXs ${ }^{\text {sc }}$ alone significantly downregulated Nox2 expression (versus vehicle, $p<0.05$ ). NPC-EXs ${ }^{\text {anti-miR-210 }}$ can downregulate Nox2 expression, but had much less effect than NPC-EXs ${ }^{\text {con }}$ and NPC-EXs ${ }^{\text {sc }}$ had (versus NPC-EXs ${ }^{\text {con }}$ or NPC-EXs ${ }^{\text {sc }}, p<0.05$ ). Similarly, NPC-EXs ${ }^{\text {miR-210 }}$ had the most effect on decreasing Ang IIinduced Nox2 expression in ECs.

Collectively, this data suggests that miR-210 plays a role in the protective effects of NPC-EXs on ECs against Ang II-induced oxidative stress, and exogenous miR-210 can boost the antioxidant and antiapoptosis effects elicited by NPC-EXs.

3.4. NPC-EXs ${ }^{\text {anti-miR-210 }}$ Were Less, Whereas NPC-EXs $s^{\text {miR-210 }}$ Were More Effective on Increasing VEGF Secretion and Improving the Tube Formation Ability of ECs. In order to assess whether NPC-EXs can improve the endothelial angiogenic function compromised by Ang II, we analyzed VEGF level in the culture medium and the tube formation ability of ECs. Our data showed that the level of VEGF in the culture medium was decreased in ECs injured by Ang II (versus control, $p<0.05$ ). In the treatment groups, coculture with NPC-EXs ${ }^{\mathrm{con}}$ and NPC-EXs ${ }^{\mathrm{sc}}$ increased the VEGF level (versus vehicle, $p<0.05$ ), whereas NPC$\mathrm{EXs}^{\text {anti-miR-210 }}$ had less effect than NPC-EXs ${ }^{\text {con }}$ and NPC$\mathrm{EXs}^{\mathrm{sc}}$ had (versus NPC-EXs ${ }^{\text {con }}$ or NPC-EXs ${ }^{\mathrm{sc}}, p<0.05$ ). Similarly, NPC-EXs ${ }^{\text {miR-210 }}$ had the most effect on upregulating VEGF secretion of ECs among the four types of NPC-EXs (Figure 5(a)).

Similarly, the tube formation ability of ECs was compromised by Ang II (versus control, $p<0.05$ ). Coculture with NPC-EXs ${ }^{\text {con }}$ or NPC-EXs ${ }^{\text {sc }}$ alone significantly improved the tube formation ability of ECs subjected to Ang II (versus vehicle, $p<0.05)$. NPC-EXs ${ }^{\text {anti-miR-210 }}$ displayed less effect than NPC-EXs ${ }^{\text {con }}$ or NPC-EXs ${ }^{\text {sc }}$ did. Likely, NPC-EXs ${ }^{\text {miR-210 }}$ had the most effect on promoting tube formation (Figure 5(b)). Taken together, these results indicate that miR-210 is involved in the effect elicited by NPC-EXs on improving the angiogenic function of Ang II-injured ECs. 


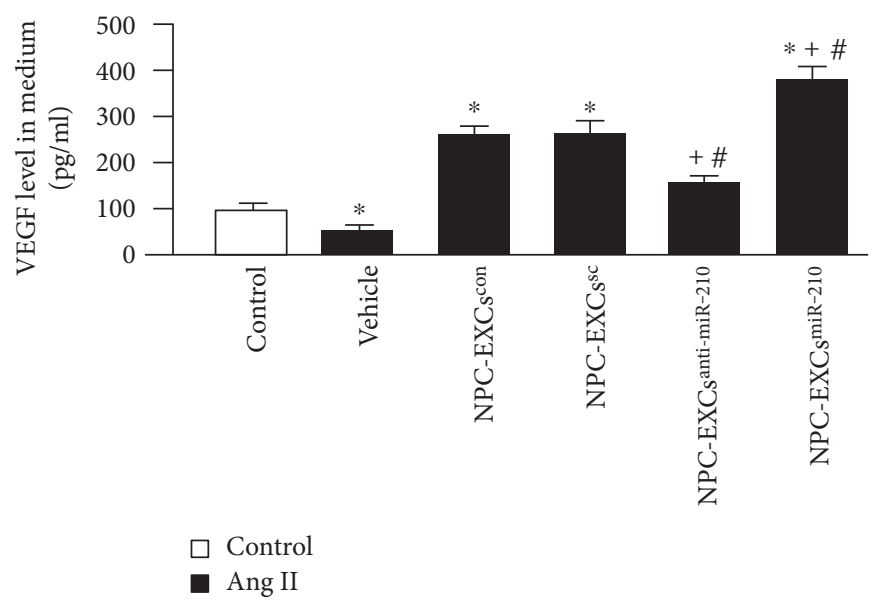

(a)

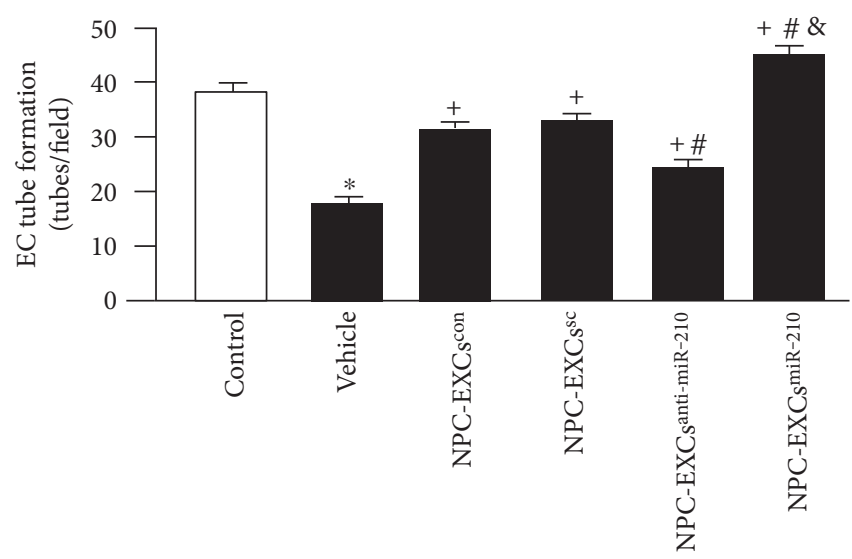

$$
\begin{aligned}
& \square \text { Control } \\
& \square \text { Ang II }
\end{aligned}
$$
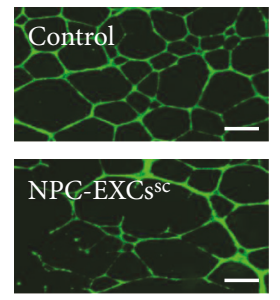
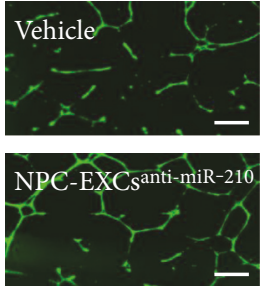

(b)

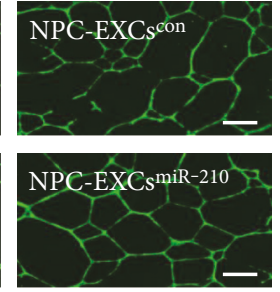

FIGURE 5: Modulation of miR-210 altered the effect of NPC-EXs on improving the VEGF secretion and tube formation ability of ECs. (a) VEGF level in the culture medium of ECs treated with various NPC-EXs. (b) Summarized data of tube formation and representative images showing the tube formation of ECs treated by various NPC-EXs; bar: $500 \mu \mathrm{m} .{ }^{*} p<0.05$ versus control; ${ }^{+} p<0.05$ versus vehicle; ${ }^{\#} p<0.05$ versus NPC-EXs ${ }^{\text {con }}$ or NPC-EXs ${ }^{\text {sc }} ;{ }^{\circledR} p<0.05$, versus NPC-EXs ${ }^{\text {anti-miR-210 }}$. Data are expressed as mean \pm SEM. $N=4 /$ group.

3.5. NPC-EXs ${ }^{\text {anti-miR-210 }}$ Were Less, Whereas NPC-EXs miR-210 $^{\text {Nite }}$ Were More Effective on Upregulating the Expression of p-VEGFR2/VEGFR2 in ECs. As shown in Figure 6, the ratio of p-VEGFR2/VEGFR2 in ECs was decreased by Ang II (versus control, $p<0.05)$. In the treatment groups, coculture with NPC-EXs ${ }^{\text {con }}$ or NPC-EXs ${ }^{\text {sc }}$ increased the phosphorylation of VEGFR2 (versus control or vehicle, $p<0.05)$. NPC-EXs ${ }^{\text {anti-miR-210 }}$ also raised the expression ratio of $\mathrm{p}$-VEGFR2/VEGFR2, but the effect was less than NPC-EXs ${ }^{\text {con }}$ or NPC-EXs ${ }^{\text {sc }}$ had (versus NPC-EXs ${ }^{\text {con }}$ or
NPC-EXs $\left.s^{\mathrm{sc}}, p<0.05\right)$. Similarly, NPC-EXs ${ }^{\mathrm{miR}-210}$ had the strongest effect on upregulating the ratio of p-VEGFR2/ VEGFR2 in ECs subjected to Ang II injury (versus NPC$\mathrm{EXs}^{\mathrm{con}}, \mathrm{NPC}-\mathrm{EXs}^{\mathrm{sc}}$, or NPC-EXs $\left.{ }^{\text {anti-miR-210 }}, p<0.05\right)$.

\section{Discussion}

In the present study, we have demonstrated that NPC-EXs have protective effects against Ang II-induced oxidative stress, cell death, and angiogenic dysfunction in ECs, which 


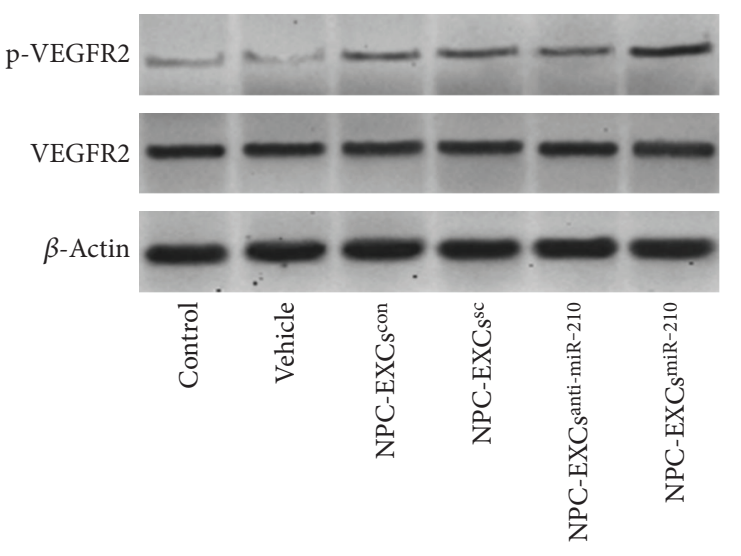

(a)

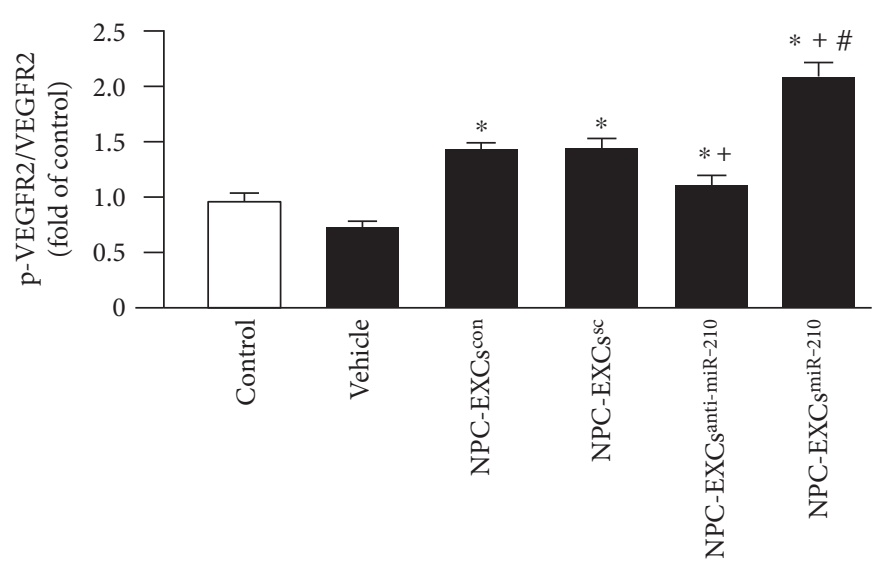

$\square$ Control

(b)

FIGURE 6: Up- and downregulation of miR-210 altered the effect of NPC-EXs on the expression of p-VEGFR2/VEGFR2 in ECs. (a) Representative western blot bands. (b) Summarized data. ${ }^{*} p<0.05$ versus vehicle; ${ }^{+} p<0.05$ versus NPC-EXs ${ }^{\text {con }}$ or NPC-EXs ${ }^{\text {sc }}$; ${ }^{*} p<0.05$ versus NPC-EXs ${ }^{\text {anti-miR-210 }}$. Data are expressed as mean \pm SEM. $N=4$ /group.

are at least partly through Nox2/ROS and VEGF/VEGFR2 signaling pathways modulated by miR-210.

Oxidative stress is the major cause of endothelial dysfunction which is implicated in various vascular diseases [31]. Ang II has been well documented to trigger ROS overproduction and decrease the production/bioavailability of nitric oxide of ECs, which consequently contribute to endothelial dysfunction [32, 33]. In this study, we used Ang II to construct an EC oxidative stress model. As agreed with previous reports [27, 34], Ang II resulted in increasing of apoptosis, overproduction of ROS, and impairment of angiogenic function (tube formation ability and VEGF secretion), along with the upregulation of Nox2 level and downregulation of the ratio of $\mathrm{p}$-VEGFR2/ VEGFR2 in ECs.

We previously demonstrated that NPCs could decrease apoptosis and ROS overproduction on hypoxia/reoxygenation-injured ECs [9]. Our study is supported by a recent report showing that neural crest-derived stem cells promote the survival of neurons under normal and oxidative stress conditions in a mutant neuron cell line [7]. However, the underlying mechanism requires further investigation. Currently, EXs are emerging as cell-cell communicators. Mounting evidence shows that stem cell-derived EXs harbor the benefits of their parent cells and can alter the function of recipient cells $[13,16,17]$. For instance, mesenchymal stem cell-derived extracellular vesicles can enhance cell survival in kidney injury [17] and promote functional recovery and neurovascular plasticity after stroke in rats [13]. We have demonstrated that endothelial progenitor cell-derived vesicles can protect ECs against hypoxia/reoxygenation [16]. In this study, we investigated whether NPC-EXs could rescue injured ECs following direct oxidative stress by using the Ang II injury model. In order to test our hypothesis, we cocultured NPC-EXs ${ }^{\text {con }}$ with Ang II-injured ECs and found that NPC-EXs ${ }^{\text {con }}$ could be uptaken by ECs and can attenuate Ang II-induced apoptosis, ROS overproduction, and Nox2 upregulation. In addition, the tube formation capacity and VEGF secretion capacities compromised by Ang II were also rescued by $\mathrm{NPC}-\mathrm{EXs}^{\mathrm{con}}$. All of these findings suggest that NPC-EXs can protect ECs against Ang II, associated with the suppression of Nox2 expression and subsequently inhibiting ROS overproduction.

Interestingly, we also found that the level of miR-210 was increased in ECs after coincubation with NPC-EXs. This raised the hypothesis that the protective effects of NPC-EXs on Ang II-induced ECs might be related with miR-210. Previous studies have demonstrated that miR-210 is ubiquitously expressed in a wide range of cells and displays versatile functions such as antioxidative stress $[15,25,35,36]$ and apoptosis defense [20-22]. Blockade of miR-210 in myoblasts greatly increases myoblast cell sensitivity to oxidative stress and mitochondrial dysfunction [25]. Overexpression of miR-210 enhances the survival of mesenchymal stem cells in an oxidative stress environment [35] and reduces ROS overproduction as well as cardiomyocyte death in response to hypoxia-reoxygenation [36]. Recently, Wang et al. reported that EXs derived from inducible pluripotent stem cells can deliver miR-210 to cardiomyocytes and protect them against $\mathrm{H}_{2} \mathrm{O}_{2}$-induced oxidative stress [15]. In order to investigate the potential mechanisms, we tested our hypothesis that miR-210 modulates the beneficial effects exhibited by NPC-EXs on Ang II-injured ECs. First, we upor downregulated miR-210 in NPCs and found that the miR-210 level was parallelly raised or reduced in their corresponding EXs, NPC-EXs ${ }^{\text {miR-210, }}$ and NPC-EXs ${ }^{\text {anti-miR-210. }}$ This suggests that NPCs carried by miR-210 was packaged into NPC-EXs. Next, we cocultured NPC-EXs ${ }^{\text {miR-210 }}$, NPC$\mathrm{EXs}^{\text {anti-miR-210 }}$, and their controls (NPC-EXs ${ }^{\mathrm{sc}}$ ) with Ang IIinjured ECs. Our data showed that both NPC-EXs ${ }^{\mathrm{sc}}$ and NPC-EXs ${ }^{\text {miR-210 }}$ increased the miR-210 level in ECs. Meanwhile, we found that the protein level of ephrin A3, a target gene of miR-210 [19], was significantly downregulated by NPC-EXs ${ }^{\text {miR-210 }}$ in Ang II-injured ECs. These results reflect 
that NPC-EXs can deliver miR-210 into ECs. Besides, we found that NPC-EXs ${ }^{\text {miR-210 }}$ had the strongest effects on decreasing Ang II-induced apoptosis and ROS production, indicating miR-210 can boost the protection effects of NPC-EXs on ECs. As one of the most prominent sources of vascular ROS [37], Nox2 level was also evaluated. We found the Nox2 level in ECs was remarkably decreased by NPC-EXs ${ }^{\text {miR-210 }}$, suggesting that the antioxidant effect of NPC-EXs might be associated with the Nox2/ROS signal. In contrast, NPC-EXs ${ }^{\text {anti-miR-210 }}$ partially reduced the protective effects of NPC-EXs, which further confirmed the important role of miR-210 in NPC-EXs for protecting ECs from Ang II-induced injury. This data also suggests that other cargoes such as proteins and cytokines carried by NPC-EXs might also participate in the protection event.

As we know, VEGF is a proangiogenic factor [38] which could be modulated by ephrin A3 [39]. In the present study, our data indicated that NPC-EXs ${ }^{\text {miR-210 }}$ could decrease ephrin A3 level whereas increase VEGF level in the culture medium of ECs, suggesting that ephrin A3 is connecting miR-210 and VEGF in ECs. The result showing the increase of VEGF level is coherent with that NPC-EXs ${ }^{\text {miR-210 }}$ improved tube formation ability of ECs compromised by Ang II. VEGFR2 is responsible for most downstream angiogenic effects of VEGF [40]. Binding of VEGF to VEGFR2 can activate downstream survival and migration pathways involving PI3-kinase/Akt and focal adhesion kinase, respectively [41]. In order to determine whether the increased level of VEGF can activate VEGFR2 signal, we used Western blots to evaluate the changes in the signaling proteins VEGFR2 and its phosphorylation. We found the total expression of VEGFR2 in ECs was not changed after NPC-EX treatment, while the expression of p-VEGFR2 was remarkably increased by NPC-EXs ${ }^{\mathrm{miR}-210}$, indicating the activation of the VEGF/VEGFR2 signal. Meanwhile, our data indicated that NPC-EXs $s^{\text {anti-miR-210 }}$ also upregulated the phosphorylating VEGFR2, though at a less extent as compared to that of NPC-EXs ${ }^{\text {con }}$ or NPC-EXs ${ }^{\text {sc }}$ had. This effect could be resulted from other cargoes such as proteins and cytokines carried by NPC-EXs ${ }^{\text {anti-miR-210 }}$. Collectively, these data reflect that miR-210 can modulate the effect of NPC-EXs on ECs subjected to Ang II injury by activating the VEGF/VEGFR2 signal.

\section{Conclusion}

Our results demonstrate that miR-210 can modulate the effects of NPC-EXs on protecting ECs from Ang II-induced oxidative stress, majorly through the Nox $2 /$ ROS and VEGF/ VEGFR2 signals.

\section{Conflicts of Interest}

There is no conflict of interests.

\section{Authors' Contributions}

Hua Liu, Jinju Wang, and Yusen Chen contributed equally to this work.

\section{Acknowledgments}

This work was supported by the American Heart Association (16SDG26420078), the Natural Science Foundation of Hubei Province (2015CFA084), the Outstanding Young Scientific and Technological Innovation Team in the Colleges and Universities of Hubei Province, China (T201523), and the Scientific Research Project supported by the Wuhan Sports University (2014QZ07, 2016XH24).

\section{References}

[1] Y. Ohara, T. E. Peterson, and D. G. Harrison, "Hypercholesterolemia increases endothelial superoxide anion production," The Journal of Clinical Investigation, vol. 91, no. 6, pp. 25462551, 1993.

[2] S. Grunfeld, C. A. Hamilton, S. Mesaros et al., "Role of superoxide in the depressed nitric oxide production by the endothelium of genetically hypertensive rats," Hypertension, vol. 26, no. 6 Part 1, pp. 854-857, 1995.

[3] S. Rajagopalan, X. P. Meng, S. Ramasamy, D. G. Harrison, and Z. S. Galis, "Reactive oxygen species produced by macrophagederived foam cells regulate the activity of vascular matrix metalloproteinases in vitro. Implications for atherosclerotic plaque stability," The Journal of Clinical Investigation, vol. 98, no. 11, pp. 2572-2579, 1996.

[4] J. Bauersachs, A. Bouloumie, D. Fraccarollo, K. Hu, R. Busse, and G. Ertl, "Endothelial dysfunction in chronic myocardial infarction despite increased vascular endothelial nitric oxide synthase and soluble guanylate cyclase expression: role of enhanced vascular superoxide production," Circulation, vol. 100, no. 3, pp. 292-298, 1999.

[5] M. Aoki, T. Nata, R. Morishita et al., "Endothelial apoptosis induced by oxidative stress through activation of NF- $\kappa$ B: antiapoptotic effect of antioxidant agents on endothelial cells," Hypertension, vol. 38, no. 1, pp. 48-55, 2001.

[6] N. Marui, M. K. Offermann, R. Swerlick et al., "Vascular cell adhesion molecule-1 (VCAM-1) gene transcription and expression are regulated through an antioxidant-sensitive mechanism in human vascular endothelial cells," The Journal of Clinical Investigation, vol. 92, no. 4, pp. 1866-1874, 1993.

[7] T. Aggarwal, J. Hoeber, P. Ivert, S. Vasylovska, and E. N. Kozlova, "Boundary cap neural crest stem cells promote survival of mutant SOD1 motor neurons," Neurotherapeutics, vol. 9, pp. 1-11, 2017.

[8] M. Tavazoie, L. Van der Veken, V. Silva-Vargas et al., "A specialized vascular niche for adult neural stem cells," Cell Stem Cell, vol. 3, no. 3, pp. 279-288, 2008.

[9] J. Wang, Y. Chen, Y. Yang et al., "Endothelial progenitor cells and neural progenitor cells synergistically protect cerebral endothelial cells from hypoxia/reoxygenation-induced injury via activating the PI3K/Akt pathway," Molecular Brain, vol. 9, no. 1, p. 12, 2016.

[10] Y. Shao, Y. Shen, T. Chen, F. Xu, X. Chen, and S. Zheng, "The functions and clinical applications of tumor-derived exosomes," Oncotarget, vol. 7, no. 37, pp. 60736-60751, 2016.

[11] Y. Chen, Y. Tang, W. Long, and C. Zhang, "Stem cell-released microvesicles and exosomes as novel biomarkers and treatments of diseases," Stem Cells International, vol. 2016, Article ID 2417268, 2 pages, 2016. 
[12] N. Tang, B. Sun, A. Gupta, H. Rempel, and L. Pulliam, "Monocyte exosomes induce adhesion molecules and cytokines via activation of NF- $\kappa \mathrm{B}$ in endothelial cells," The FASEB Journal, vol. 30, no. 9, pp. 3097-3106, 2016.

[13] H. Xin, Y. Li, Z. Liu et al., "miR-133b promotes neural plasticity and functional recovery after treatment of stroke with multipotent mesenchymal stromal cells in rats via transfer of exosome-enriched extracellular particles," Stem Cells, vol. 31, no. 12, pp. 2737-2746, 2013.

[14] K. Wu, Y. Yang, Y. Zhong et al., "The effects of microvesicles on endothelial progenitor cells are compromised in type 2 diabetic patients via downregulation of the miR-126/VEGFR2 pathway," American Journal of Physiology. Endocrinology and Metabolism, vol. 310, no. 10, pp. E828-E837, 2016.

[15] Y. Wang, L. Zhang, Y. Li et al., "Exosomes/microvesicles from induced pluripotent stem cells deliver cardioprotective miRNAs and prevent cardiomyocyte apoptosis in the ischemic myocardium," International Journal of Cardiology, vol. 192, pp. 61-69, 2015.

[16] J. Wang, S. Chen, X. Ma et al., "Effects of endothelial progenitor cell-derived microvesicles on hypoxia/reoxygenation-induced endothelial dysfunction and apoptosis," Oxidative Medicine and Cellular Longevity, vol. 2013, Article ID 572729, 9 pages, 2013.

[17] S. Bruno, C. Grange, F. Collino et al., "Microvesicles derived from mesenchymal stem cells enhance survival in a lethal model of acute kidney injury," PloS One, vol. 7, no. 3, article e33115, 2012.

[18] Y. Gong, F. Xu, L. Zhang et al., "MicroRNA expression signature for Satb2-induced osteogenic differentiation in bone marrow stromal cells," Molecular and Cellular Biochemistry, vol. 387, no. 1-2, pp. 227-239, 2014.

[19] P. Fasanaro, Y. D'Alessandra, V. Di Stefano et al., "MicroRNA210 modulates endothelial cell response to hypoxia and inhibits the receptor tyrosine kinase ligand ephrin-A3," The Journal of Biological Chemistry, vol. 283, no. 23, pp. 1587815883, 2008.

[20] H. W. Kim, H. K. Haider, S. Jiang, and M. Ashraf, "Ischemic preconditioning augments survival of stem cells via miR-210 expression by targeting caspase-8-associated protein 2," The Journal of Biological Chemistry, vol. 284, no. 48, pp. 3316133168, 2009.

[21] S. Y. Chan and J. Loscalzo, "MicroRNA-210: a unique and pleiotropic hypoxamir," Cell Cycle, vol. 9, no. 6, pp. 10721083, 2010.

[22] C. Devlin, S. Greco, F. Martelli, and M. Ivan, "miR-210: more than a silent player in hypoxia," IUBMB Life, vol. 63, no. 2, pp. 94-100, 2011.

[23] Y. Jiang, L. Li, X. Tan, B. Liu, Y. Zhang, and C. Li, "miR-210 mediates vagus nerve stimulation-induced antioxidant stress and anti-apoptosis reactions following cerebral ischemia/ reperfusion injury in rats," Journal of Neurochemistry, vol. 134, no. 1, pp. 173-181, 2015.

[24] J. H. Kim, S. G. Park, S. Y. Song, J. K. Kim, and J. H. Sung, "Reactive oxygen species-responsive miR-210 regulates proliferation and migration of adipose-derived stem cells via PTPN2," Cell Death \& Disease, vol. 4, no. 4, p. e588, 2013.

[25] L. Cicchillitti, V. Di Stefano, E. Isaia et al., "Hypoxia-inducible factor 1-alpha induces miR-210 in normoxic differentiating myoblasts," The Journal of Biological Chemistry, vol. 287, no. 53, pp. 44761-44771, 2012.
[26] J. Wang, R. Guo, Y. Yang et al., "The novel methods for analysis of exosomes released from endothelial cells and endothelial progenitor cells," Stem Cells International, vol. 2016, Article ID 2639728, 12 pages, 2016.

[27] X. Xiao, C. Zhang, X. Ma et al., “Angiotensin-(1-7) counteracts angiotensin II-induced dysfunction in cerebral endothelial cells via modulating Nox $2 /$ ROS and PI3K/NO pathways," Experimental Cell Research, vol. 336, no. 1, pp. 58-65, 2015.

[28] E. C. Jensen, "Quantitative analysis of histological staining and fluorescence using ImageJ," The Anatomical Record, vol. 296, no. 3, pp. 378-381, 2013.

[29] H. Xia, S. Suda, S. Bindom et al., "ACE2-mediated reduction of oxidative stress in the central nervous system is associated with improvement of autonomic function," One, vol. 6, no. 7, article e22682, 2011.

[30] T. G. Chen, J. Z. Chen, and X. X. Wang, "Effects of rapamycin on number activity and eNOS of endothelial progenitor cells from peripheral blood," Cell Proliferation, vol. 39, no. 2, pp. 117-125, 2006.

[31] H. Ogita and J. Liao, "Endothelial function and oxidative stress," Endothelium, vol. 11, no. 2, pp. 123-132, 2004.

[32] M. Sata and D. Fukuda, "Crucial role of renin-angiotensin system in the pathogenesis of atherosclerosis," The Journal of Medical Investigation, vol. 57, no. 1-2, pp. 12-25, 2010.

[33] C. E. Murdoch, S. P. Alom-Ruiz, M. Wang et al., "Role of endothelial Nox2 NADPH oxidase in angiotensin II-induced hypertension and vasomotor dysfunction," Basic Research in Cardiology, vol. 106, no. 4, pp. 527-538, 2011.

[34] S. Sivasinprasasn, R. Pantan, S. Thummayot, J. Tocharus, A. Suksamrarn, and C. Tocharus, "Cyanidin-3-glucoside attenuates angiotensin II-induced oxidative stress and inflammation in vascular endothelial cells," Chemico-Biological Interactions, vol. 28, 2016.

[35] J. Xu, Z. Huang, L. Lin et al., "miR-210 over-expression enhances mesenchymal stem cell survival in an oxidative stress environment through antioxidation and c-Met pathway activation," Science China. Life Sciences, vol. 57, no. 10, pp. 989-997, 2014.

[36] R. K. Mutharasan, V. Nagpal, Y. Ichikawa, and H. Ardehali, "MicroRNA-210 is upregulated in hypoxic cardiomyocytes through Akt- and p53-dependent pathways and exerts cytoprotective effects," American Journal of Physiology. Heart and Circulatory Physiology, vol. 301, no. 4, pp. H1519H1530, 2011.

[37] R. P. Brandes, N. Weissmann, and K. Schroder, "NADPH oxidases in cardiovascular disease," Free Radical Biology \& Medicine, vol. 49, no. 5, pp. 687-706, 2010.

[38] J. Skog, T. Wurdinger, S. Van Rijn et al., "Glioblastoma microvesicles transport RNA and proteins that promote tumour growth and provide diagnostic biomarkers," Nature Cell Biology, vol. 10, no. 12, pp. 1470-1476, 2008.

[39] Z. Wang, Z. Liu, B. Liu, G. Liu, and S. Wu, "Dissecting the roles of ephrin-A3 in malignant peripheral nerve sheath tumor by TALENs," Oncology Reports, vol. 34, no. 1, pp. 391-398, 2015.

[40] T. Sakurai and M. Kudo, "Signaling pathways governing tumor angiogenesis," Oncology, vol. 81, Supplement 1, pp. 24-29, 2011.

[41] G. Taraboletti, S. D'Ascenzo, I. Giusti et al., "Bioavailability of VEGF in tumor-shed vesicles depends on vesicle burst induced by acidic pH," Neoplasia, vol. 8, no. 2, pp. 96-103, 2006. 


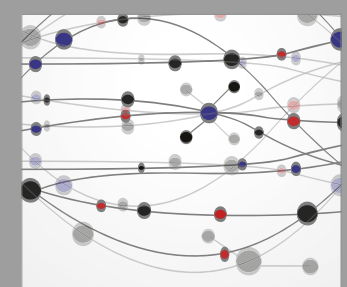

The Scientific World Journal
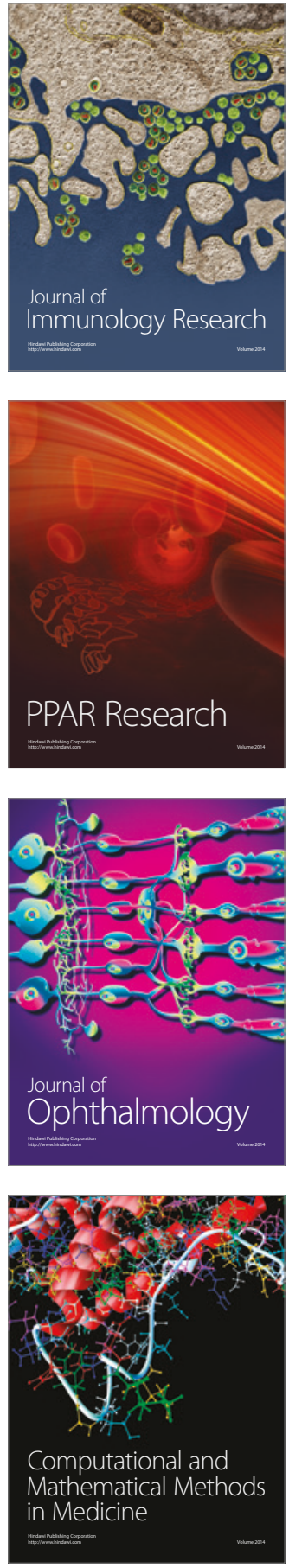

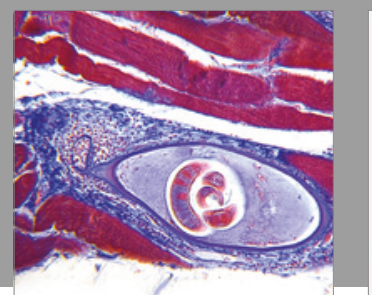

Gastroenterology Research and Practice
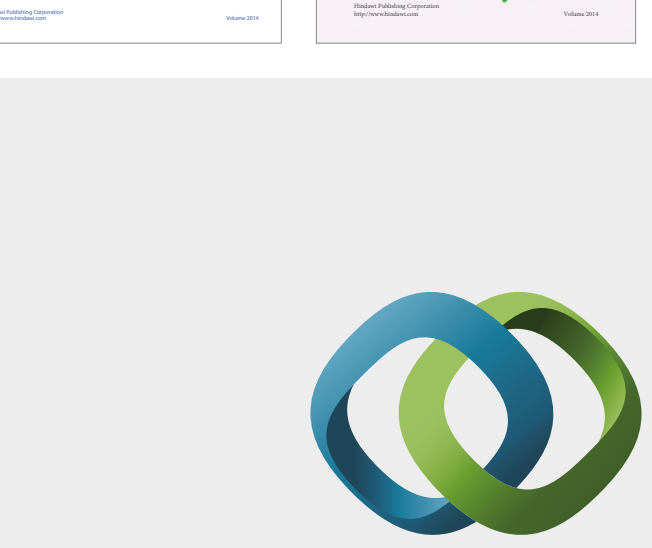

\section{Hindawi}

Submit your manuscripts at

https://www.hindawi.com
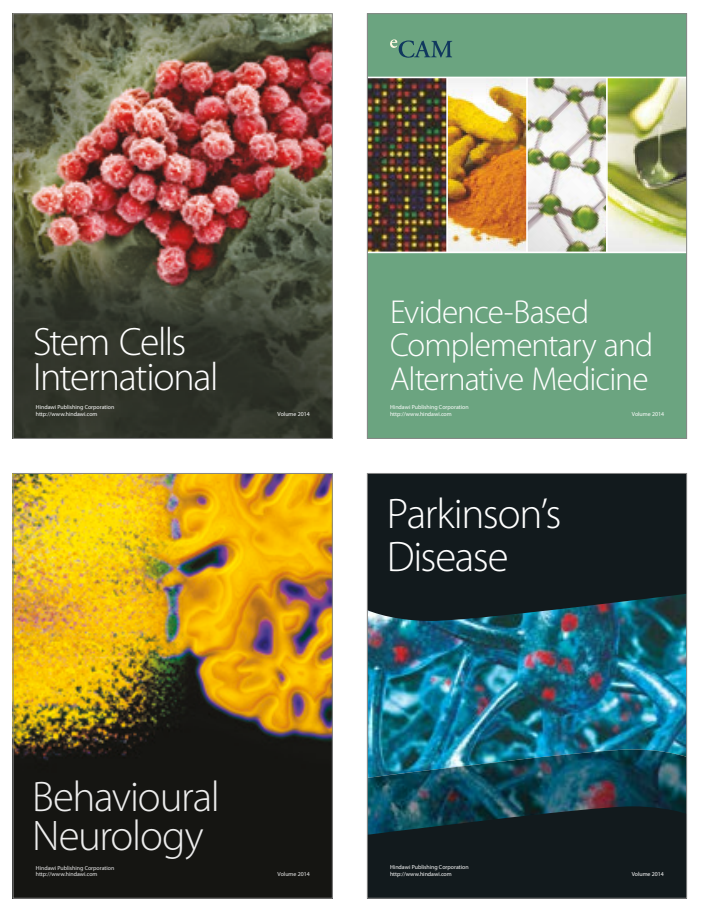
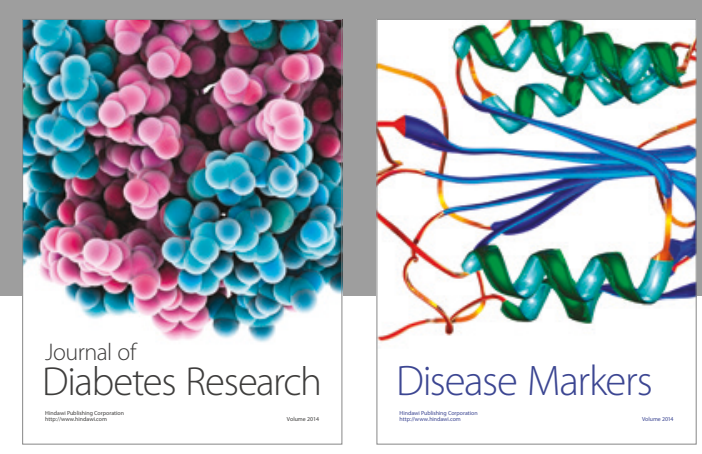

Disease Markers
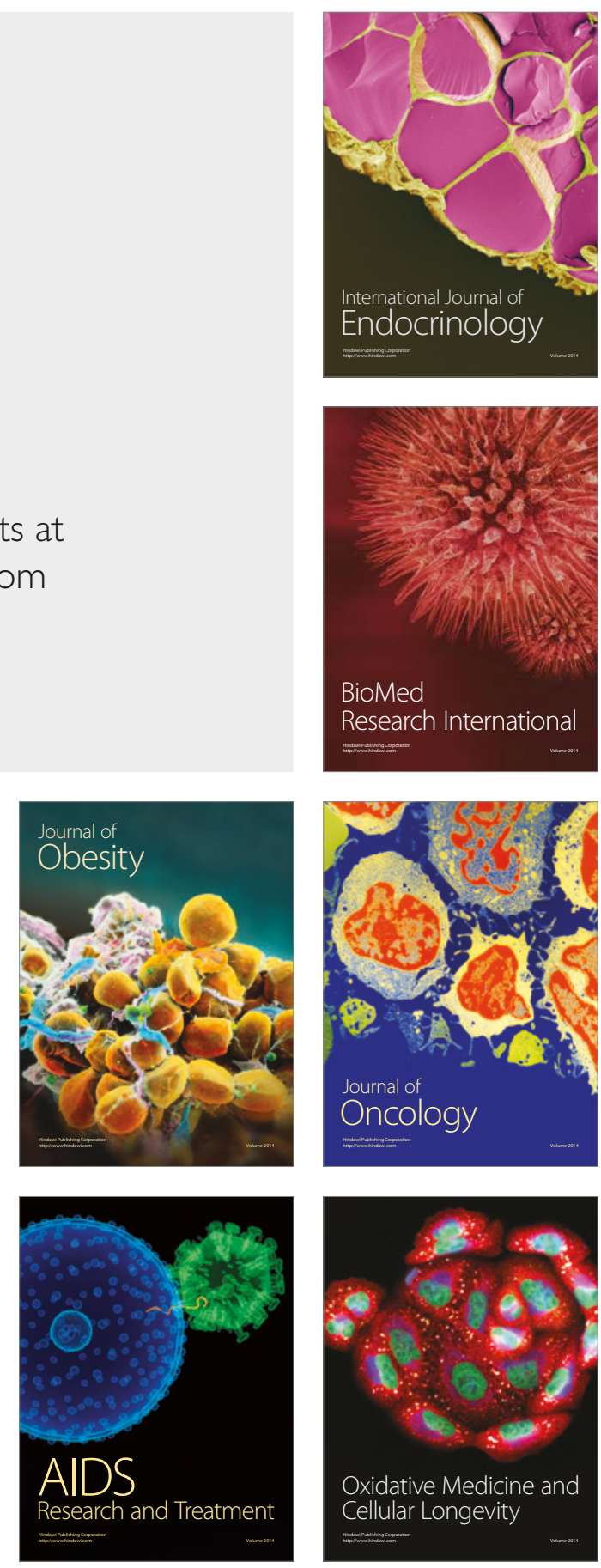